\title{
A Non-Spatial Reality
}

\author{
Massimiliano Sassoli de Bianchi ${ }^{1,2}$
}

Published online: 1 November 2020

(C) The Author(s) 2020

\begin{abstract}
It is generally assumed, and usually taken for granted, that reality is fully contained in space. However, when taking a closer look at the strange behavior of the entities of the micro-world, we are forced to abandon such a prejudice and recognize that space is just a temporary crystallization of a small theatre for reality, where the material entities can take a place and meet with each other. More precisely, phenomena like quantum entanglement, quantum interference effects and quantum indistinguishability, when analyzed attentively, tell us that there is much more in our physical reality than what meets our three-dimensional human eyes. But if the building blocks of our physical reality are non-spatial, what does it mean? Can we understand what the nature of a non-spatial entity is? And if so, what are the consequences for our view of the world in which we live and evolve as a species? This article was written having in mind one of the objectives of the Center Leo Apostel for Interdisciplinary Studies, that of a broad dissemination of scientific knowledge. Hence, it addresses a transversal audience of readers, both academic and nonacademic, hoping to stimulate in this way the interdisciplinary dialogue about foundational issues in science.
\end{abstract}

Keywords Quantum theory · Non-spatiality · Entanglement · Interference effects · Indistinguishability $\cdot$ Conceptuality interpretation

\section{Introduction}

According to Plato's famous allegory of the cave, we are like prisoners chained from time immemorial in a dark cave, only watching and studying flickering shadows on a wall, believing that those shadows, and the surface of the wall, are all that exists in our reality. In Plato's allegory, one distinguishes two levels: the empirical or spatiotemporal level, which is that of the appearances, and the ontological level, considered to be that part of the world that remains unperceived by our ordinary senses but somehow could be understood by our intellect. In other words, the ontological level is that of the "real entities," whereas the empirical level is that of the "appearances of these same real entities." To put it differently, following Plato's allegory, higher-dimensional entities, having a "deeper" reality, would

Massimiliano Sassoli de Bianchi

msassoli@vub.ac.be; autoricerca@gmail.com

1 Center Leo Apostel for Interdisciplinary Studies, Brussels Free University, Krijgskundestraat 33, 1160 Brussels, Belgium

2 Laboratorio Di Autoricerca Di Base, Via Cadepiano 18, 6917 Barbengo, Switzerland 
Fig. 1 Abbott's allegory of Flatland here exemplified as the middle-world defined by the twodimensional surface of a lake

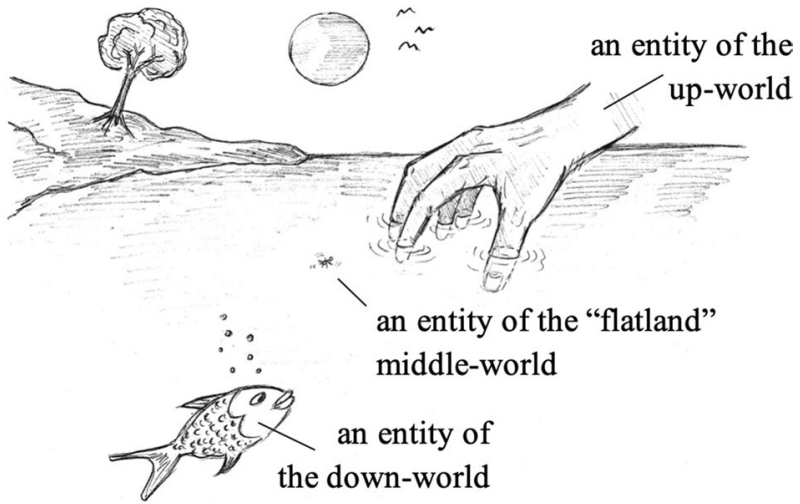

exist, casting all sorts of shadows onto the lower-dimensional "wall" of our humanly constructed spatial (or spatiotemporal) representation.

A similar allegory was conveyed by the English schoolmaster Edwin A. Abbott, in his "Romance in Many Dimensions" (Abbott 1884), written to criticize the Victorian culture. According Abbott's allegory (which was famously used by Carl Sagan in his 1980s "Cosmos" TV series, to explain the difficulties we have in visualizing a world of four dimensions), we are pretty much like the residents of a Flatland, i.e., low-dimensional beings living in a "thin layer" of a much vaster reality; a layer that is constantly traversed by entities of higher dimensionality that we cannot perceive in their fullness.

As an example, imagine a lake in a beautiful spring day. Its surface defines three distinct worlds. There is the down-world, rather thick, populated by three-dimensional aquatic creatures such as fish; there is the up-world, more rarefied, also populated by three-dimensional creatures, like birds; and there is the "flatland middle-world," as defined by the very surface of the lake, a reality of an intermediate density populated by essentially two-dimensional creatures, like small wingless insects that never leave the thin film of water. ${ }^{1}$

According to Abbott, we humans are somehow like the flat creatures of this middleworld, with all the perceptual (and cognitive) disadvantages it entails. Imagine being one of the insects that lives at the boundary between the up-world and the down-world, not knowing being at the frontier of realities of higher dimensionality, as you always lived in a two-dimensional environment, with a two-dimensional body, and you cannot directly experience a third dimension, or a fourth, a fifth, etc. (see Fig. 1).

Then, suppose that a three-dimensional entity of the up-world, say a human hand, dips its five fingers into the water. From the limited perspective of a middle-world creature, you will see appearing out of nowhere five strange entities, more or less spherical, that for just a moment will manifest in your space (see Fig. 2). Surely, you will mistake those ephemeral traces for genuinely two-dimensional individual entities, completely separated and independent from one another. Nonetheless, from the perspective of a three-dimensional hyper-entity of the up-world, it is clear that those five spherical-entities are not separate, but interconnected: they are part of a unitary three-dimensional entity and they only appear separate when their higher dimensional structure is viewed from the limited perspective of a two-dimensional representation.

\footnotetext{
1 Of course, strictly speaking, the small wingless insects living on the surface of the lake are still threedimensional entities, so our example must be understood in an ideal sense, thinking of the insects on the surface as genuine two-dimensional beings, likewise the inhabitants of Flatland, in Abbott's novella.
} 
Fig. 2 The inhabitants of a twodimensional world might mistake a single whole three-dimensional entity, like a hand, as five separated entities, moving one independently of the others

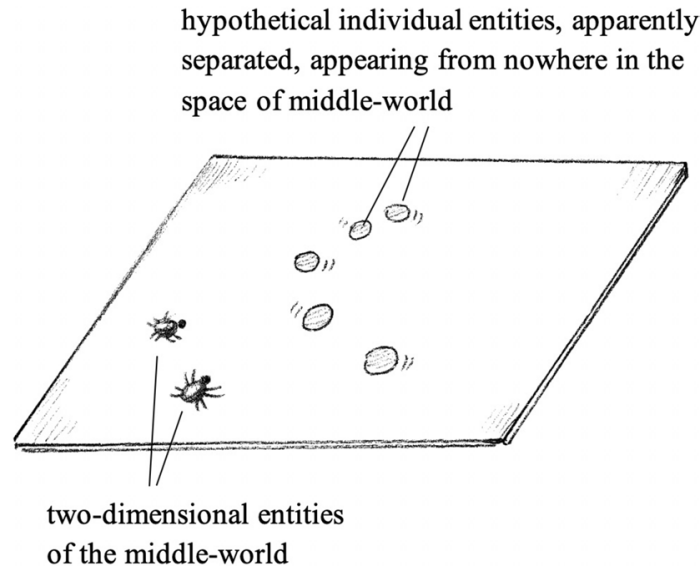

What we will try to do in this article, is to explain why Plato and Abbott, in their bewildering allegories, had a correct perception about our reality, when sensing that a lot was indeed happening "behind the scenes" of our spatial representation, without our knowledge. But we will also try to indicate what Plato and Abbott were not able to guess: the nature of the entities populating these "behind the scenes" of our spatial representation, and how they can relate with the latter.

Now, if it is true that space (and more generally spacetime) is not the container of our physical reality, but only a specific theater in which a very parochial representation is taking place, the first thing we must address is how we came to consider such representation in the first place, then mistaking it for everything that exists. In other words: How did we construct our spatial theater?

This has to do with the fact that, as a species, we have evolved since hundreds of thousands of years in a very particular niche of our reality: that of the surface of our beautiful planet Earth. As Sagan used to say, contemplating the picture of our planet taken in 1990 by the Voyager 1 space probe, from a distance of about 6 billion kilometers, "Earth is a very small stage in a vast cosmic arena." This is certainly true when we compare our planet with the immense spatial "cosmic dark" in which it is immersed. But our planet, and the entities with which we have interacted at its surface by means of our dense bodies, are also in turn "vast cosmic arenas," if we compare them with the socalled microscopic "particles" forming them. By this we mean that we have been surrounded by quite some particular physical entities, of a macroscopic size, and from our multiple interactions with these entities, which we experienced by means of our dense human bodies in a rather hot environment, we started a long time ago the construction of a prototypical worldview, in the attempt to order our experiences into a consistent map of relations.

From this pre-cultural and pre-scientific construction, a first clothing and decoration of reality resulted, allowing us to identify those portions of it that were recognizable as aggregates of sufficiently stable properties, where by 'stable' we mean that the properties characterizing these aggregates could remain actual for long enough to become easy to observe. These aggregates of relatively permanent properties (think of a piece of matter having a given size, weight, temperature, etc.) were what we today call, in physics, classical entities, or macroscopic objects, or simply objects, bodies, etc., which also 
include the astronomical bodies we can see moving in the sky, like the Moon and the Sun, obeying with good approximation the laws of non-relativistic classical mechanics.

One can distinguish two different fundamental directions of penetration in our pre-cultural process of clothing and decoration of reality (Aerts and Aerts 2004). One direction, which we have just mentioned, is a penetration in depth, through which we have initially identified those phenomena that, according to our senses, particularly those of sight and touch (Aerts 2014), stood out compared to others, because of their availability in interacting with our bodies and becoming part of our experiences, and also because such availability persisted long enough, so allowing us to have multiple experiences with them. In other terms, by means of our penetration in depth of reality, we have recognized the existence of experientially separated and stable portions of it.

The second, in a sense complementary direction of penetration, can be called penetration in width. It corresponds to our effort to organize and order the content of our experiences with all these different aggregates of stable properties, i.e., with the different physical objects that appeared to us to be separated, in the sense of not being part of a same aggregate and not influencing each other in a significant way. This process of penetration in width, through which we have identified the more important and evident relations among these entities of our ordinary experiences, can be understood as an ordering process giving rise to a space. And since our practical experiences were essentially with classical entities, the space of relations that emerged is what we call today the three-dimensional Euclidean space.

In other terms, space can be essentially considered as a specific theater of reality that emerged when a given set of experiences was properly ordered and organized, i.e., put in relation to each other (Aerts and Aerts 2004). The reason why such a specific theater of reality has been mistaken over time for a fundamental substantive container for the latter (a position still maintained today by many if not the majority of scientists) is easy to understand: as time went by, we have simply forgot about our construction, and since the typology of our experiences remained basically the same, it was easy and natural to start believing that all of our reality would necessarily fit into such theater, so that the theater and its content, and reality, would just be one and the same thing. This belief, however, becomes difficult (if not impossible) to maintain in our days as following the discoveries of modern physics genuinely new experiences were accessed, in controlled experimental contexts, with entities behaving very differently from those discovered in our initial process of 'penetration in depth', and which rather stubbornly did not lend themselves to be included, or fully included, in the relational space that was built thus far.

\section{Entanglement and Non-Spatiality}

A paradigmatic example of the breakdown of our Euclidean spatial theater construction is the discovery of quantum entanglement. At the theoretical level, it was initially discussed by Einstein, Podolsky and Rosen (1935) and by Schrödinger (1935), and its existence has now been firmly established in numerous experiments, starting from the historical ones performed with photons, in 1982, by the French group of Alain Aspect (Aspect et al 1982; Aspect 1999).

In a nutshell, two entities are in an entangled state if they can be spatially separated by arbitrary distances and yet remain invisibly interconnected, so that they are able to influence each other or behave as if they were a single entity. Well, to say it all, the notion of 
entanglement in quantum theory does not depend in any way on whether the two entities are spatially separated or not, but it is certainly when this is the case that the truly nonordinary aspect of the "entanglement relationship" becomes evident.

The reason why entanglement is incompatible with our Euclidean construction is very simple to understand. As we said, during our penetration in width of reality we have constructed a spatial representation of the different possible relations between the entities that we could identify. In this representation, the notion of spatial distance was also used to describe the degree of experimental separation between entities, in the sense that the greater their spatial separation the lesser their possible mutual influences (principle of locality). Now, for two entities to be experimentally separated, let us call them entity $A$ and entity $B$, it means that when we test a property on entity $A$ the outcome of the test will not depend (in an ontological sense) on other tests we may perform (simultaneously or in a sequential way) on entity $B$, and vice versa (Aerts 1984). For ordinary classical/spatial entities this is guaranteed whenever the distance separating them is sufficient to guarantee that no signal can have the time to propagate between them to possibly influence the outcomes of their respective tests, before their execution. And more generally, this is guaranteed whenever there is no 'third element of reality' that would connect the two entities in some way. And of course, if such connecting element would be present and detectable, we would not say anymore that the two entities are spatially separated, but that they form a single interconnected whole.

So, spatial separation and experimental separation were in a sense considered to be synonyms, as the former was precisely used to characterize the latter, during the construction of our Euclidean theater. But let us now explain how entanglement is revealed in experiments conducted in the physics' laboratories. This is done by analyzing possible correlations resulting from the execution of joint measurements (i.e., joint observations) on composite systems. This is however a subtle issue as also entities that are experimentally separated can have some of their properties strongly correlated. For this, it is sufficient that they were once connected in their past and were subsequently disconnected by some physical process, in such a way that the process of disconnection created correlations.

It was the great merit of the Northern Irish physicist John Bell to have proposed specific inequalities, nowadays called Bell inequalities (Bell 1964, 1971), only involving quantities that are experimentally accessible, able to test if the observed correlations were already existing prior to the joint measurements, hence were only discovered by the latter, or if the correlations were only potential prior to the measurement, hence were literally created by the latter.

The Belgian physicist Diederik Aerts proposed to call the former correlations of the first kind and the latter correlations of the second kind, being those of the second kind which are typical of quantum entanglement (Aerts 1990). More precisely, and roughly speaking, correlations of the second kind can violate Bell's inequalities, and therefore reveal the presence of entanglement, whereas correlations of the first kind cannot violate Bell's inequalities, and therefore describe a situation of experimental separation.

Let us provide a famous example of correlations of the first kind, i.e., of correlations that are not considered to be the signature of quantum entanglement: Bertlmann's socks (Bell 1981). Dr. Bertlmann was a colleague of Bell, who liked to always wear socks of different colors. Of course, it was quite unpredictable which color he would have on a given foot on a given day, but if one was able to see that the first sock was, say, pink, one could obtain immediate information about the fact that the other sock would be non-pink (see Fig. 3). Of course, no mystery here: the color of the two socks pre-existed their observation; it is not something that was created by the latter, but just discovered during it. 
Fig. 3 A depiction of Bertlmann's socks situation, as described in Bell's 1981 paper entitled "Bertlmann's socks and the nature of reality" (Bell 1981)

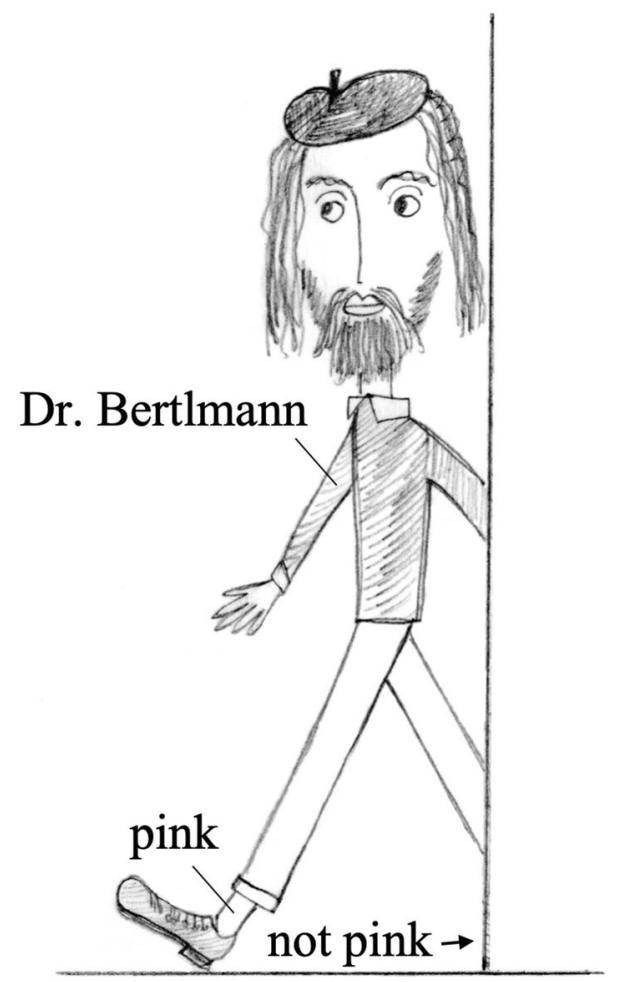

Is it also possible to provide a simple example of a system exhibiting correlations of the second kind, which are typical of quantum entanglement? The answer is affirmative. Consider an experiment where two persons (let us call them Alice and Bob, as is traditional in physics), simply hold the two ends of an unbroken elastic band of length $L$, and by pulling them they break it in two parts (Aerts 2005; Sassoli de Bianchi 2013a). This is clearly a situation where correlations are of the second kind, as the respective lengths of the two fragments are created in a genuinely unpredictable way by the joint action of Alice and Bob (see Fig. 4). However, they are always perfectly correlated, as their sum must always be equal to the length $L$ of the unbroken elastic band.

It is important to remind that quantum measurements, i.e., quantum observations, are processes that can create the very properties that are meant to be tested, i.e., observed, as in general they are only potential prior to their observation. As a consequence, when observations are performed in a joint way on a given system, they can create (actualize) correlations that were only potential prior to the join observation. Alice and Bob breaking an elastic band by their joint actions, creating in this way different possible correlated couples of outcomes, like $\left(d_{1}, L-d_{1}\right),\left(d_{2}, L-d_{2}\right),\left(d_{3}, L-d_{3}\right), \ldots$, is a perfect exemplification of this situation, and in fact it can be proven that the process of breaking elastic bands is able to violate Bell's inequalities (Aerts 2005; Sassoli de Bianchi 2013a; Aerts et al 2019).

To make perfectly clear the difference between a situation capable of creating significant correlations, compared to a situation where this is not the case, let us consider another simple example. Imagine that Alice and Bob hold each of them a die in their hands. If they jointly roll their die, say on a table, they will obtain a couple of upper faces, which 


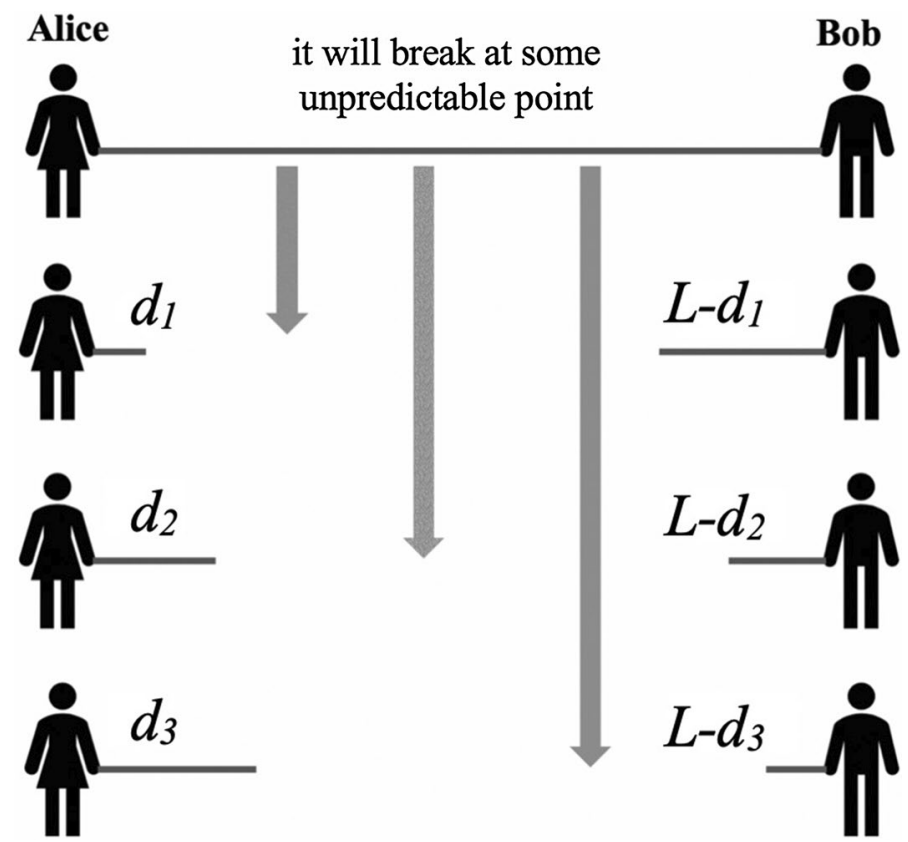

Fig. 4 Three possible outcomes of a process of breaking an elastic band. Although the obtained lengths of the two fragments can be different at each 'breaking experiment', they are also perfectly correlated, as their sum is always equal to the total length $L$ of the unbroken elastic

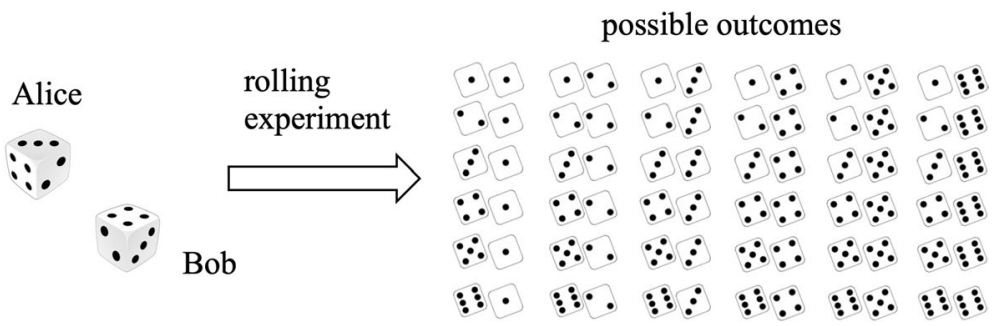

Fig. 5 By rolling two unconnected dice, 36 equiprobable pairs of different upper face outcomes can be obtained. Even though for each dice an upper face is created in an unpredictable way, the outcomes obtained for each die are completely independent of one another, hence no correlations are created by the joint rolling experiment and Bell's inequalities cannot be violated

correspond to the outcome of their joint "rolling experiment." Assuming that the two dice are not rigged, and considering that they are experimentally separated entities, not influencing each other in whatsoever way, 36 different couples of outcomes will be obtained with equal probability (see Fig. 5). This is clearly a situation where there are no correlations.

Imagine now that the two dice are connected in some way, so as to form an "entangled double-die system." This can be done by linking them through space by means of a rigid rod, whose two ends are attached at the center of two of their opposed faces, as indicated in Fig. 6. Then, the presence of the rod only allows Alice and Bob to roll their 


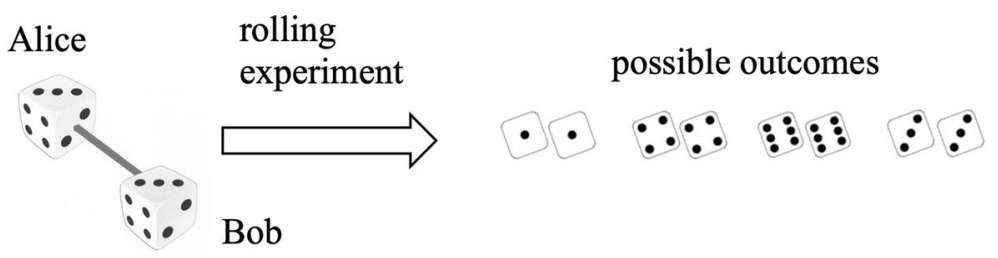

Fig. 6 By connecting the two dice, only 4 equiprobable pairs of upper face outcomes can be actualized. Hence, correlations are created by the joint rolling experiments and Bell's inequalities will be violated

die along a same direction, perpendicular to the rod, so that this time only 4 pairs of upper face outcomes can be obtained (see Fig. 6). So, we now have a process where correlations are created in an unpredictable way, and this is again a situation where Bell's inequalities will be violated (Sassoli de Bianchi 2013b, 2014).

The above examples should not be considered just as useful didactical tools: they also allow to get rid of the misconception that a violation of Bell's inequalities would only be specific to the micro-world (Aerts et al 2000; Aerts and Sassoli de Bianchi 2018). Classical macroscopic entities can also violate them, if the joint experiments performed by Alice and Bob are able to actualize potential correlations, which will generally be the case if the composite entity on which they act forms a whole, because its component parts are connected in some way. In the case of the unbroken elastic band, the connection is provided by the very substance of the elastic, and in the case of the two dice by the rigid rod. These connections are elements of reality that we can represent and detect within our three-dimensional Euclidean space. In other words, they are spatial connections.

Here comes the fundamental difference between two dice entangled by means of a rigid rod, or two elastic fragments entangled within an unbroken elastic band, and the situation of two entangled micro-entities, like say two entangled electrons, or two entangled photons. Indeed, in the latter cases the connections that create the correlations remain hidden, not only because mathematically speaking they cannot be represented in a 3-dimensional space, but also because, experimentally speaking, there is nothing detectable in the space between two spatially separated entangled micro-entities. Despite of that, the two entities can still give rise to correlations of the second kind or behave as if they would form a single whole entity and, in this way, give the impression that they can influence each other at a distance.

Einstein famously described this puzzling situation as a "spooky action at a distance" and physicists nowadays use for it the term of non-locality. However, if quantum entanglement expresses a form of connectivity that does not happen in space, i.e., that is the consequence of the presence of non-spatial connections, the correct term to be used is not non-locality, but non-spatiality. And this means that microscopic entities would generally not be in space but be brought in space only when interacting with macroscopic entities like the measuring apparatuses, or when forming macroscopic aggregates in standard conditions (Aerts 1999). And since entangled states are the vast majority of states, we have to conclude that the vast majority of our physical reality would be non-spatial.

It should be said, however, that it remains nowadays an uncommon view to consider that Bell's inequalities violations would be the consequence of correlations of the second kind, even though the latter are implicit in the quantum formalism. This means that the majority of physicists still consider that to explain these violations one needs to evoke some kind of influence-like mechanism. However, if influences are assumed to propagate in space, then 
they have to do so at a superluminal speed, and one can show that such speed must exceed that of light by at least four orders of magnitude (Salart et al 2008; Cocciaro et al 2011).

This possibility of superluminal influences is usually considered acceptable because quantum mechanics, in its standard formulation and interpretation, is protected from possible conflicts with relativistic causality by the so-called no-signaling conditions on quantum correlations (also called marginal laws), stating that the quantum probabilities have to obey certain specific relations, precisely preventing Alice and Bob to use their statistical data to communicate with one another at an effective superluminal speed. ${ }^{2}$ However, in spite of the no-signaling conditions, a more attentive analysis shows that correlations resulting from influences propagating in space at a superluminal finite speed can always be exploited for obtaining faster-than-light communications (Coretti et al 2011; Bancal et al 2012). So, to use the words of the Swiss physicist Nicolas Gisin and collaborators (Bancal et al 2012):

If we want to keep no-signalling, it shows that quantum non-locality must necessarily relate discontinuously parts of the universe that are arbitrarily distant. This gives further weight to the idea that quantum correlations somehow arise from outside spacetime, in the sense that no story in space and time can describe how they occur.

In other words, an explanation of quantum correlations in terms of influences propagating in space, at some speed, seems to lead to an incurable conflict with relativity and to open the door to temporal paradoxes. So, either one remains in the uncomfortable position of not having an explanation for the entanglement phenomenon, or one accepts that it has to do with non-spatiality and with correlations of the second kind, that is, correlations relaying to a common cause which is not yet actual at the moment of a joint measurement but is actualized by it in an unpredictable way.

\section{Superposition and Non-Spatiality}

One might wonder at this point if non-spatiality would be an aspect of our physical reality that can only emerge when entities interact together and, as a result of their interaction, enter in a so-called entangled state. In other words, when electrons are not entangled, but in so-called separable states, can we consider them again as pure spatial entities, behaving as particles or waves (depending on the experimental context)?

To see that even this view is untenable, let us focus on a very specific property of quantum micro-entities, their spin, which is usually (although improperly) described as an intrinsic angular momentum, allowing the spinorial entity to behave like a micro-magnet (a magnetic dipole, with a north and south pole). There are many reasons why this image cannot be considered to be correct, and one is that the rotation should then be so swift that, if the micro-entity is considered to be a corpuscle with a given radius, its periphery would have to move at superluminal speed, in violation of the relativistic limit. But let us nevertheless consider that it would be possible to associate a direction in space to each spin state of an electron (or a neutron, etc.) i.e., that the idea that a spin would be a spatiallike property, describable as a micromagnet having a given orientation in space, would be essentially correct [regarding the problematic notion of spins' directions, see also Aerts

\footnotetext{
${ }^{2}$ See however Aerts et al (2019), for a general approach showing that a violation of the no-signaling conditions, in addition to Bell's inequality, is to be expected in joint quantum measurements, without this necessarily implying that a superluminal communication would be possible.
} 
Fig. 7 A sketch of the LLL silicon (Si) crystal interferometer, able to split the incident beam into four distinct beams, which are then detected by the four detectors $D_{1}, D_{2}, D_{3}$ and $D_{4}$. Along the path of one of the internal beams, a well localized static magnetic field $B$ is applied, so as to rotate the neutron's spin of an angle that is proportional to the intensity of the magnetic field

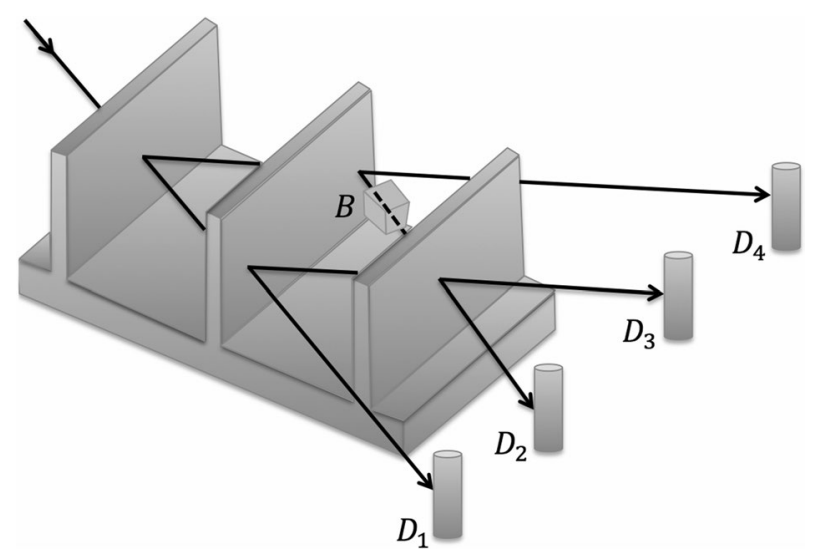

and Sassoli de Bianchi (2015a). Then, in the same way a magnetic dipole, when rotated $360^{\circ}$ (i.e., $2 \pi$ ), is brought back to the same state, one would expect that when the spin of an electron is rotated $2 \pi$, it is also brought back to the exact same state. But this is not what happens and in fact one needs to rotate a spin of $4 \pi\left(720^{\circ}\right)$ for it to be back to the same state.

This is not just a theoretical hypothesis but an experimental fact that has been verified in some beautiful experiments conducted in the mid-seventies of last century, not with electrons but with neutrons. Let us explain the rationale of these experiments, as they reveal a lot about the genuine non-spatial nature of micro-entities like neutrons, in a way that is totally independent from the previously described phenomenon of entanglement. Indeed, these experiments were performed using a single neutron at a time, which was made to interfere with itself in a way that corpuscular or wave-like entities are definitely unable to do.

These fundamental experiments were conducted by the groups of the Austrian physicist Helmut Rauch and of the American physicist Samuel A. Werner (Rauch et al 1975; Werner et al 1975), using a so-called LLL device made from a single Si-crystal [for a theoretical and conceptual analysis of these celebrated experiments, see Sassoli de Bianchi (2017)]. As described in Fig. 7, this is a monolithic device consisting of three perfect crystal plates that are cut from a large and perfect $S i$-crystal. The size of the crystal is typically of $7 \mathrm{~cm}$ and the thickness of its three plates is less than half a centimeter.

What is important to observe for our discussion is that the speed of the incoming (ultracold) neutrons, and the distance between them, was such that on average there was typically just a single neutron at a time passing through the device. Now, because of the geometry and orientation of the three parallel crystal plates, each time a neutron encountered one of them, it could only move along two distinct paths: one corresponding to the neutron being simply transmitted through the plate, without being deviated, and the other with the neutron being deflected by a given angle, due to the specific geometry of the internal structure of the crystal. In other words, at each encounter with a plate, there was a bifurcation, where the neutron could only take two different possible paths.

As described in Fig. 7, this means that following the interaction with the three plates, a neutron could exit the LLL device along four possible distinct paths, with its presence being revealed by the corresponding four detectors $D_{1}, D_{2}, D_{3}$ and $D_{4}$. Two of these paths exit the device without interacting with the third plate (corresponding to detectors $D_{1}$ and 
Fig. 8 The data obtained by Rauch et al (1975), showing the typical $4 \pi$-periodicity of the intensity measured at detector $D_{2}$, when the strength of the applied magnetic field $B$, located on the upper internal path, is varied, so as to vary correspondingly the rotation angle of the neutron's spin, according to the phenomenon known as Larmor precession

\section{Intensity at detector $D_{2}$}

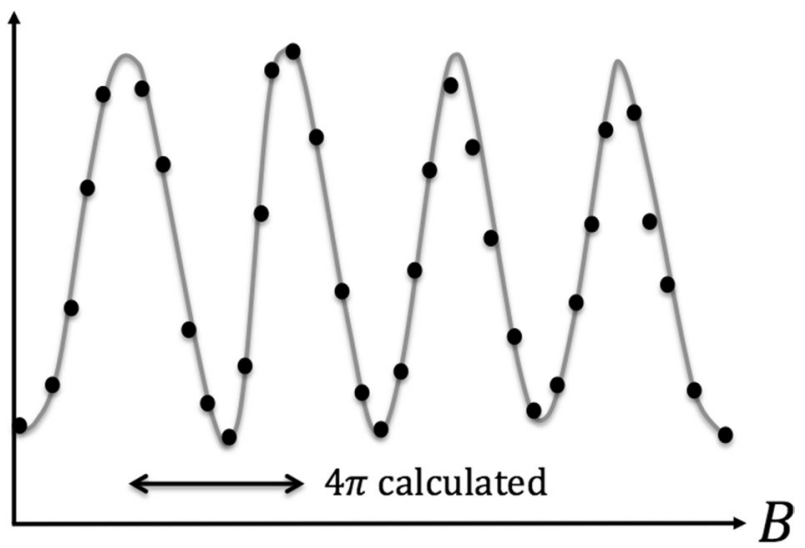

$D_{4}$ ) whereas the other two recombine (i.e., superpose) exactly at the level of the third plate, finally also exiting the crystal towards detectors $D_{2}$ and $D_{3}$.

The idea of the experiment was to place a well localized (static) magnetic field along one of the two internal paths, so as to rotate the neutron's spin passing through it of a given angle, proportional to the intensity of the applied magnetic field. If neutrons (entering and exiting the LLL crystal one at a time) would just follow one, and only one, of the possible paths, the presence of the magnetic field would then not be able to affect the probabilities with which the different detectors click. However, since two of the internal paths recombine at the level of the third plate, quantum mechanics predicts that the associated probability amplitudes have to superpose, and since the action of the magnetic field is to shift the phase of the corresponding amplitude, a phenomenon of interference is expected to occur, proportionally to the rotation of the neutron's spin as induced by the magnetic field (things are in fact a bit more complicate and we refer to Sassoli de Bianchi (2017) for a more complete discussion).

Remarkably, what was observed, in accordance with the predictions of the theory, is that the intensity measured at the detectors $D_{2}$ and $D_{3}$ exhibited a $4 \pi$-periodicity with respect to the spin rotation angle (see Fig. 8), thus demonstrating that only following a $720^{\circ}$ rotation a neutron's spin entity is brought exactly into the same state, which is a property impossible to associate to any spatial entity like those we interact with in our everyday life.

The experimental highlighting of this unusual $4 \pi$-symmetry of a neutron's spin, instead of the $2 \pi$-symmetry of an ordinary spatial object, as surprising and spectacular as it is, is certainly not the most amazing aspect that was evidenced in these experiments, when properly analyzed. To explain what we mean, it is useful to rescale the LLL crystal up to 25 million times and project it onto the European map [see also the discussion in Aerts (1999) and Sassoli de Bianchi (2017)]. As can be seen on Fig. 9, neutrons then pass through the first plate in France, close to Paris, and once they have also traversed the second plate, the northern path crosses Denmark and Sweden, whereas the southern path goes over Poland and Lithuania, before both paths recombining in Latvia.

What is important to observe is that the neutrons used in these interferometry experiments have a so-called (longitudinal) coherence length that is typically of one millionth of a centimeter. When such length is scaled up 25 million times, one finds that the spatial region within which these imaginary (rescaled) giant neutrons can be acted upon, 


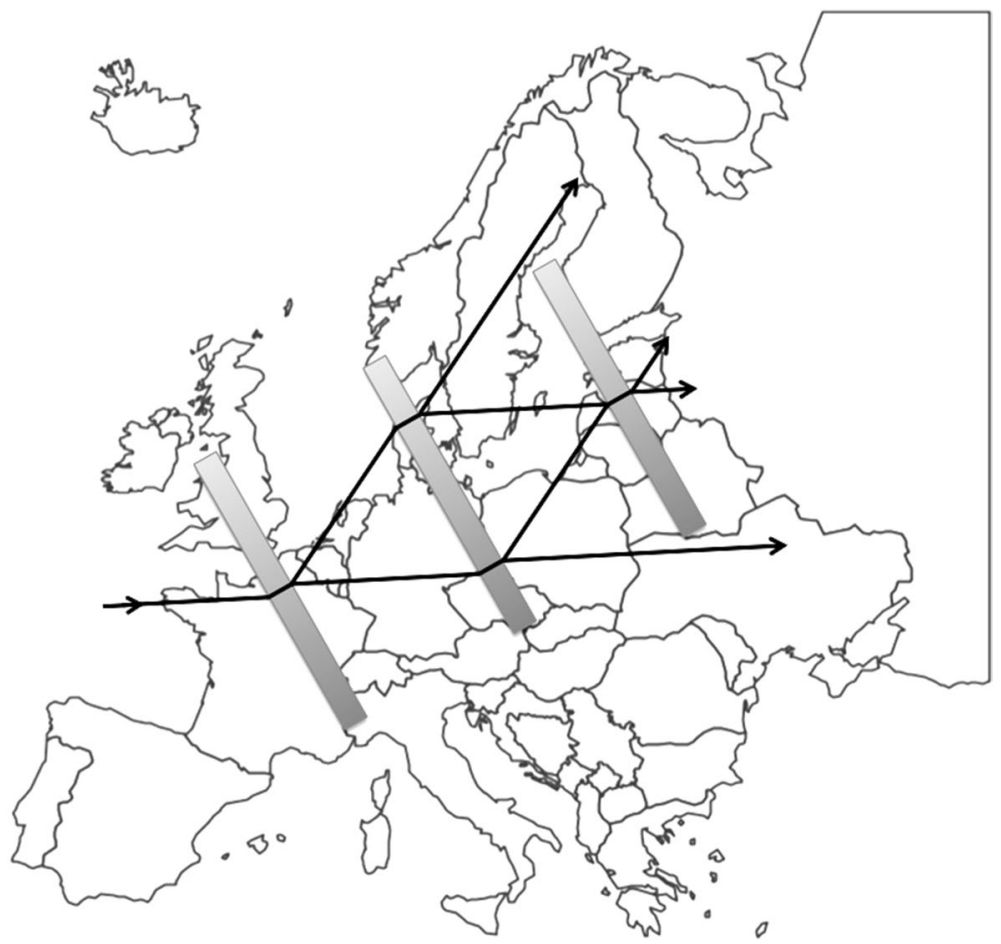

Fig. 9 A bird view of the LLL device, here scaled up 25 million times and projected on the European map

when they travel along their different possible paths, is a small cube of $25 \mathrm{~cm}$ ! Comparing this with the hundreds of kilometers distance separating Sweden from Poland, it is clear that there is no way to interpret the neutrons entering the LLL device as extended spatial objects (as it would be the case if they were waves): they are truly more like small projectiles moving along very narrow paths.

But if this would be the case, then the $4 \pi$-symmetry of a neutron's spin could not be observed, as it requires the amplitudes coming from the two different paths, one traversing the localized magnetic field in Sweden, and the other one going over Poland (and thus not traversing the magnetic field) to superpose and interfere. If a neutron would truly be like a localized projectile, considering that just a single neutron enters the device at a time, then either it would encounter the magnetic field, if it takes the northern path, or it would not encounter it, if it takes the southern path. But in none of these two situations interference effects would be observed and the $D_{2}$ and $D_{3}$ detectors would just click on average the same number of times.

Consequently, we cannot say that a neutron is like a spatial well-localized corpuscle, moving on a single path, nor can we say that it is like an extended entity spread out in space, because its coherence length is very small and it can only be detected along very narrow paths, and never in the space between them. On the other hand, a neutron is able to jointly explore, or "sense," the different possible paths at the same time, something that a genuine spatial entity is obviously unable to do. In other words, these remarkable neutron interferometry experiments really force us to go beyond the wave-particle duality and 
accept that micro-entities, like neutrons, have a non-spatial nature, i.e., can be in non-spatial states, which however does not imply that they would not be influenced by spatial local apparatuses. $^{3}$

Let us mention that in more recent times superposition experiments have been successfully performed also using much more complex entities than neutrons, like large molecules, thanks to the advent of more advanced matter-wave interferometers and techniques for obtaining slow macromolecular beams. For instance, Gerlich et al (2011) were able to put molecular entities composed of 430 atoms (covalently bound together) into superposition states with respect to the "left arm" and "right arm" of their interferometer, with a path separation of about two orders of magnitude larger than the size of these molecules. Similar experiments were performed by Sandra Eibenberger and collegues, in Vienna, obtaining genuine quantum superposition states for giant molecules containing over 800 atoms (Eibenberger et al 2013). All these experiments clearly show that the internal complexity of an entity that is brought in a state of spatial superposition is not at all affected by the process of delocalization, hence the idea that a superposition state would be akin to a 'spreading of the entity in a wave-like pattern over space' cannot be considered to be correct.

So, the question arises: How can we even imagine entities of this kind? Let us provide an example that the author heard the first time from Constantin Piron, when teaching his famous course of quantum mechanics in Geneva (Piron 1990; Sassoli de Bianchi 2017). Take a $10 €$ bill (the original example was with 10 Swiss francs). When it is intact, we can certainly say that the $10 €$ are located somewhere in space, like it is the case for an ordinary classical entity. More precisely, the location of the $10 €$ is exactly the location of the $10 €$ bill. But what happens when the bill is torn in two parts and the obtained pieces are spatially separated (see Fig. 10)?

Clearly, when this happens, we cannot say anymore that the $10 €$ are still located somewhere in space, although we cannot even say that they would have completely disappeared from space. Imagine for a moment that the two bill's fragments are placed into two different boxes. In a sense, we can say that the $10 €$ are present in the two boxes, but it is also true that they are contained in none of them, which is very similar to the situation of a neutron that, in a sense, is simultaneously present in all its different possible paths within an interferometer, although it is also present in none of them. More precisely, when taken together, the two boxes certainly contain the $10 €$, but only in potential terms, which can become actual $10 €$ only in the moment the two fragments are taken out from the boxes and joined together.

\footnotetext{
3 Rauch's experiment alone cannot exclude the possibility of a spatial explanation à la de Broglie-Bohm, in terms of a wave plus a particle, both having a full physical reality; see for instance Vigier et al 1987. In an approach of this kind, the particle element is considered to be always perfectly localized in a specific path of the interferometer, whereas in the other path only a so-called empty wave would be travelling, the presence of which would then explain the interference effects. However, the hypothesis of empty waves was shown to be inconsistent in experiments performed by Mandel and collaborators (Zou et al 1992) and, generally speaking, approaches based on three-dimensional pilot waves will be unable to explain higher order (multiparticle) coherence effects. So, strictly speaking, Rauch's experiment provides support to the non-spatiality hypothesis only if additional experiments are also considered, eliminating alternative pilot wave-based (spatial) explanations. Note that Bohm himself was aware that a pure spatial picture of a pilot wave guiding the movement of particles would face serious problems when dealing with more than a single entity, as the quantum potential guiding their movement will then no-longer act in a three- dimensional Euclidean space, but in a configuration space of higher dimension (Bohm 1957).
} 


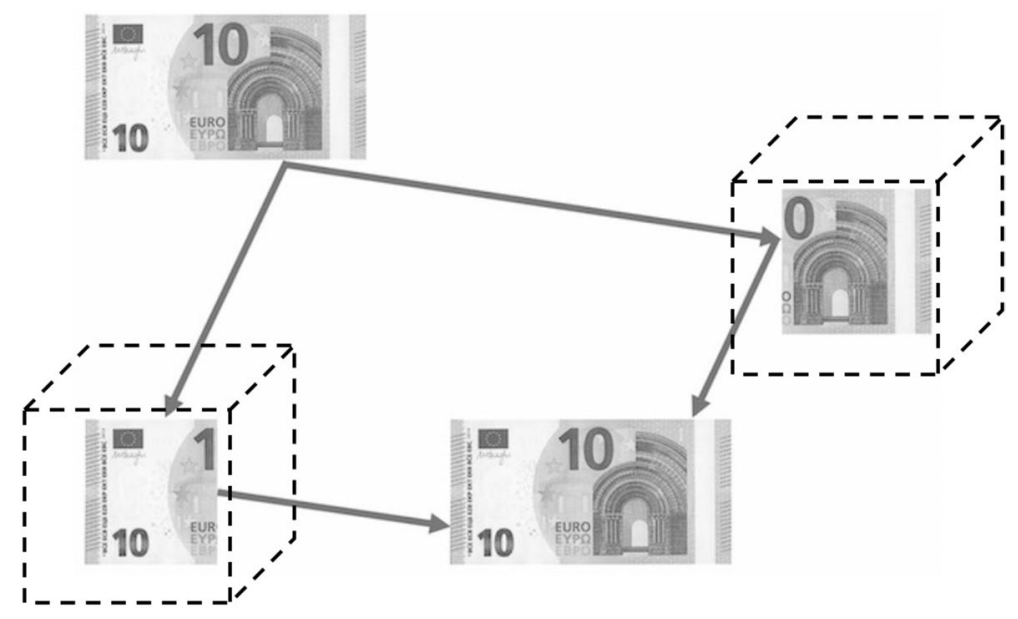

Fig. 10 A $10 €$ bill is first torn apart, then the two bill's fragments are moved on different paths (and for instance inserted into two separated boxes), to ultimately recombine at some other place in space. In the process, the $10 €$ disappear from our spatial theater, to reappear again when the full bill is reconstituted

This is of course only a metaphor, but a very revealing and interesting one, as it carries two crucial ideas. As we mentioned already, there is the idea that so-called quantum nonlocality would be an expression of non-spatiality, where by the latter term one should not understand that a non-spatial entity would have necessarily totally disappeared from our spatial theater, as if this would be the case then it would become impossible to understand why a quantum (micro) entity can be easily influenced by classical (macro) entities, like the measurement apparatuses we use in the physics' laboratories, which are certainly stably present in space. In other words, micro-entities, like neutrons, although non-spatial, maintain a specific relation with space, in the sense that they always remain available in being detected inside of it, with a degree of availability that varies depending on the locations and their state of preparation. In the experiment with the neutron interferometer, there are only narrow paths where a neutron has a very high degree of availability in being "sucked" in space, whereas the degree of availability is very low for the regions in-between these paths. The latter are therefore sort of "interdimensional windows" through which one can act on neutrons and access their non-spatial reality.

Now, the reason why the $10 €$ example works well with our intuition is that $10 €$ are not just an object: they are also a concept. What we mean to say is that one has to distinguish the $10 €$ conceptual entity from the $10 €$ bill objectual entity. The $10 €$, as a conceptual entity, can be instantiated (that is, concretized, objectified) in many different ways. A full $10 €$ bill is a possibility, but also 5 coins of $2 €$ is a possible way of instantiating $10 €$, so using metal instead of paper, and of course we can also have $10 €$ instantiated in an electronic way, as a specific transfer appearing in a given bank account. What is important to observe, in the case of the paper bill, is that when we affirm that the $10 €$ are in the two separated boxes, and at the same time in none of them, this statement makes sense because two different $10 €$ notions are jointly present in our mind. On one hand, there is the $10 €$ bill, which is a concrete object, and as such is certainly not present in any one of the two single boxes (as just a piece of it is present in each box). On the other hand, there are the 10 $\epsilon$ understood as a more abstract conceptual entity, which as we observed can be instantiated in our spatial theater in many different ways. When we say that the $10 €$ are simultaneously 

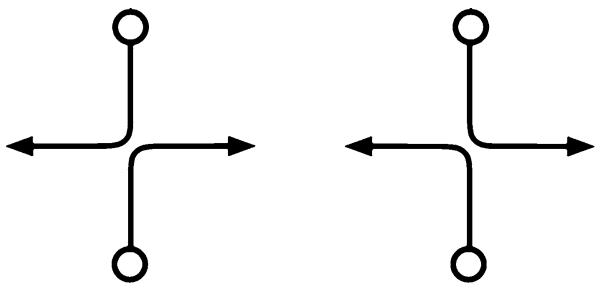

Fig. 11 The situation of two identical billiard balls directed towards each other with equal speed, one from the North and the other from the South. Since they move in opposite directions, they will do the same after the collision and their speeds will also again be equal. If the collision is somewhat off-center, each ball will be deviated from its original direction of motion by some angle. Here two situations are described: on the left, the ball coming from North is deviated to the West, and consequently the ball coming from South is deviated to the East, whereas on the right it is the other way around. Clearly, only by discerning the trajectories of the two balls, during their collision, it is possible to know the direction towards which each one of them has been diverted to, hence distinguish the two situations. For quantum micro-entities, because of the absence of a notion of spatial trajectory, these two situations cannot anymore be distinguished

in the two boxes, we are more specifically referring to the $10 €$ as an abstract entity that can be instantiated in different ways, in different contexts.

So, could it be that an entity like a neutron would be able to behave in the way it behaves because it is similar in nature to a human concept? In other words, could it be that the non-spatiality of the micro-entities is telling us that they would be like conceptual entities, which can manifest in different states, some very concrete, like those we can associate with spatial properties, and some more abstract, which we cannot associate any longer with spatial properties, and that this would explain their otherwise inexplicable behavior? Note that we are not saying here that quantum entities would be human concepts, what we are saying is that they would share with the latter a same conceptual nature, similarly to how an electromagnetic wave and a sound wave, although very different physical entities, do share a same undulatory nature (Aerts et al 2018a, b, c).

\section{Indistinguishability and non-spatiality}

Before spending some words on the possibility of ascribing a conceptual nature to the entities forming our physical reality, let us mention another quantum "strangeness," also directly pointing to the non-spatiality of quantum entities, and therefore also to their possible conceptual nature: indistinguishability. Spatial entities (think of a billiard ball) are strictly speaking always distinguishable, even when they are identical, that is, even when they possess the same set of properties (like a same mass, volume, charge, angular momentum, temperature, color, etc.). Indeed, assuming the impenetrability of two physical objects, i.e., that they cannot occupy at the same time the same location in space, it follows that they can always be distinguished by considering the different trajectories they follow in space. In a certain sense, each trajectory provides to each spatial entity a sort of label that allows one to distinguish it from the other entities having the same properties (see Fig. 11).

So, spatial material entities can be identical and at the same time also remain always distinguishable, at least in principle, because they cannot possess at a given time the same spatial properties and the latter can always be used to set them apart. If entities, like say neutrons, would be like small marbles living in space, they would be distinguishable, and their distinguishability would play a role when they are assembled together, for example 
when forming the nucleus of an atom, or a giant (and extremely dense) entity called a neutron star, resulting from the collapsed structure of a giant star. Indeed, when identical quantum entities are brought together in regimes of temperatures where quantum effects are relevant, that is, when they can enter into stable entangled states, the fact that they are indistinguishable can produce drastic differences in their collective behavior with respect to the behavior of aggregates of classical distinguishable entities.

A gas of neutrons, which belongs to the category of entities called fermions, different from a classical ideal gas made of distinguishable entities, will have for instance its pressure being only weakly dependent on temperature, instead of being directly proportional to it. Also, a gas of indistinguishable entities called bosons (like photons or helium-4 atoms), unlike a classical ideal gas is able to form a so-called Bose-Einstein condensate, when at very low temperatures the entire collection of entities truly behaves as a sort of single entity, entering in a condensate state that is tightly connected to remarkable phenomena like superfluidity (the possibility for a fluid to have zero viscosity and flow without any loss of kinetic energy).

All this is just to emphasize that indistinguishability can have remarkable effects and that these effects have been observed in laboratories. In fact, one of the experimental problems from which quantum mechanics originated, that of blackbody radiation, i.e., the problem of explaining the spectrum and intensity of the thermal radiation emitted by a non-reflective body as a function of its temperature, could only be properly addressed by considering that all photons involved in the energy exchanges are genuinely indistinguishable and therefore obey a quantum (Bose-Einstein) statistics, instead of a classical (Maxwell-Boltzmann) statistics.

More specifically, the difference between distinguishability and indistinguishability affects the statistical behavior of a collection of identical entities by affecting the way one has to count the number of their different possible configurations, which in turn depends on the fact that, when we exchange the role of two of them, this can have or not have an observable effect.

As a very simple example, take two entities $A$ and $B$. If they are distinguishable at some level, then by exchanging their role this can have observable effects. For instance, assuming that the two entities can only be in two different states, let us call them $\psi$ and $\varphi$, it is clear that the situation where $A$ is in state $\psi$ and $B$ is in state $\varphi$ is different from the situation where $A$ is in state $\varphi$ and $B$ is in state $\psi$, so that these two possibilities must be counted separately. This means that when the two entities are distinguishable, there will be 4 different possible configurations for the composite system formed by them: the two we have just mentioned plus the configurations where the two entities are both in state $\psi$ or both in state $\varphi$ (see Fig. 12).

But when the two entities are indistinguishable, we cannot say anymore that the situation where $A$ is in state $\psi$ and $B$ is in state $\varphi$ is a different situation than when $A$ is in state $\varphi$ and $B$ is in state $\psi$, because now we have $A=B$, hence these two situations, or configurations, cannot anymore be distinguished. This means that the composite system only has a total of 3 different possible states and this different way of counting is characteristic of so-called Bose-Einstein (quantum) statistics (see Fig. 12). This holds for the typology of indistinguishable entities having integer spin, called bosons, which are allowed to be all jointly in the same state. For the typology of indistinguishable entities having fractional spin, called fermions, there is the additional constraint known as Pauli's exclusion principle, saying that two entities cannot be jointly in the same state, so that just a single configuration remains in our simple example and we are in the situation where the way of counting is characteristic of so-called Fermi-Dirac (quantum) statistics (see Fig. 12). 


\begin{tabular}{|c|c|c||c|c|}
\hline$\psi$ & $A B$ & & $A$ & $B$ \\
\hline$\varphi$ & & $A B$ & $B$ & $A$ \\
\hline
\end{tabular}

\title{
Maxwell-Boltzmann
}

way of counting

Bose-Einstein
way of counting

\begin{tabular}{|l|l|l|l|}
\hline$\psi$ & $A A$ & & $A$ \\
\hline$\varphi$ & & $A A$ & $A$ \\
\hline
\end{tabular}

\author{
\begin{tabular}{c|c|c|}
\cline { 2 - 3 } Fermi-Dirac & $\psi$ & $A$ \\
\cline { 2 - 3 } way of counting & $\varphi$ & $A$ \\
\hline
\end{tabular}
}

Fig. 12 The number of possible states for a system formed by two (non-interacting) entities that can be individually in two different states, $\psi$ and $\varphi$ when a they are distinguishable individuals (spatial objects), corresponding to the Maxwell-Boltzmann way of counting; $\mathbf{b}$ they are indistinguishable individuals and are allowed to be in the same state (bosons), corresponding to the Bose-Einstein way of counting; c they are indistinguishable individuals but are not allowed to be in the same state (fermions), corresponding to the Fermi-Dirac way of counting

Micro-entities, be them fermions or bosons, are therefore certainly individuals, but mysteriously without any individual identity, as they appear to be truly and genuinely indistinguishable. This seems to go against Leibniz's famous ontological principle of the identity of the indiscernibles, stating that no two distinct entities can exactly resemble each other in all of their properties. But then, how can it be so? As we already emphasized, if we renounce considering a micro-entity as a spatial entity, then we cannot use anymore the notion of trajectory to attach a different spatial label to each member of a collection of identical entities. These however can remain different individuals because, even if totally indistinguishable, they do possess attributes that can be measured and used to count how many of them are present in a given system.

For instance, if the total electric charge of a collection of electrons is $Q$, then knowing that a single electron has an electric charge $e$, we also know that the collection contains a number $N=Q / e$ of identical electrons, and not a single electronic entity. But how can we understand then the nature of entities that are able to remain individuals and at the same time are also truly indiscernibles?

Take again the example of the $10 €$. No doubts that $10 €$ describes a collection of entities, and more precisely that collection that is obtained by considering a combination of two concepts: the concept 10 (Ten) and the concept $€$ (Euro), joined together in the combination $10 €$ (Ten Euro). It is clear that all the euros in the combination are completely identical and all exactly in the same state, i.e., all carrying exactly the same meaning and value, so that we are truly in the presence of a collection of (here Bosonic-like) indistinguishable entities, and not of a single one. In other words, in the conceptual realm, quantum indistinguishability is not at all paradoxical but perfectly self-evident. But of course, the fact that $10 €$ is a concept, and not an object, is essential for it being able to carry the (otherwise impossible to understand) quantum feature of being many and at the same time being genuinely indistinguishable. 


\section{A Conceptuality Interpretation}

Considering that the example of the $10 €$ works so well in describing both the possibility for an entity to be non-spatial and for a collection of entities to be indiscernibles, but nevertheless still remain individuals, one may wonder if this could be more than a clever metaphor and point to a deeper truth about our physical reality: that its building blocks would not be object-like, but concept-like. In other words, one may wonder if (1) quantum entities would behave similarly to human concepts because they share with them the same conceptual nature and, conversely, if (2) human concepts, as entities of a conceptual nature, would in return behave similarly to quantum entities, in the sense that quantumness and conceptuality would just be two different ways of speaking about the same reality.

Point (2) is in a sense less controversial than point (1), so let us start with it. The last two decades have seen the development of a new domain of investigation, called quantum cognition, which was pioneered by researchers like Diederik Aerts, Andrei Khrennikov, Harald Atmanspacher and collaborators; see for instance Busemeyer and Bruza (2012), Haven and Khrennikov (2013), Wendt (2015) and Aerts et al. (2013, 2016). Let us briefly explain the reasons why this field of study emerged. In the beginning of last century, during their investigation of the micro-world physicists were confronted with experimental data that were not explainable using the existing physical theories, in particular their logical and probabilistic foundations. It is precisely in their attempts of explaining the unexplainable that quantum mechanics emerged: a theory founded on a completely different (non-classical, i.e., non-Kolmogorovian) probability calculus. Something quite similar happened to cognitive scientists when they were confronted with unexpected data collected in the ambit of numerous tests conducted on groups of human participants, in order to study the probabilities characterizing their behaviors, or decision makings. Indeed, it emerged that in many circumstances human behavior would defy logic. In a nutshell, humans appear to be quite irrational.

As an example, we can describe the situation known as the conjunction fallacy, as evidenced in so-called Linda problem (Tversky and Kahneman 1983; Morier and Borgida 1984). Consider the following description of a person named Linda:

31 years old, single, outspoken and very bright. She majored in philosophy. As a student, she was deeply concerned with issues of discrimination and social justice, and also participated in antinuclear demonstrations.

Ponder then the following two statements: (1) Linda is today a bank teller; (2) Linda is today active in a feminist movement and is a bank teller. Which of these two statements appears more plausible to you? If your answer is (2), you have just fallen victim to the conjunction fallacy, as was the case for the average opinion of the tested subjects. Now, since the idea that the concomitance of two events is more probable than the occurrence of only one of them is in evident violation of the axiomatic rules of classical (Kolmogorovian) probability theory (which in turn is based on Boolean logic), experimental situations like those evidenced in Linda's problem, and many others evidencing different logical fallacies, cannot be properly addressed by the latter.

This forced researchers to find a different paradigm in order to model, in a consistent and principled way, some of the accumulated data, and surprisingly the perfect choice appeared to be quantum mechanics. Well, maybe not so surprisingly after all, considering that the latter was equipped with all the needed conceptual and mathematical tools for dealing with all sorts of deviations from classical behaviors. Indeed, as we said, quantum 
mechanics also emerged in order to describe experimental situations which could not be explained using theories based on Boolean logic and the associated Kolmogorovian probability calculus.

It would be beyond the scope of this article to tell in a convincing way the story of quantum cognition, which by the way, to avoid possible confusions, has nothing to do with the notion of quantum brain, that is, with the speculation that quantum phenomena occurring in the brain at the micro-level would play a role in the way the brain functions, particularly in relation to the manifestation of consciousness and self-consciousness. In quantum cognition, one simply observes that quantum structures can appear at some organizational level of the mental activity, in the same way that it is possible to construct macroscopic quantum machines (for instance using elastic structures with specific geometries that can break in unpredictable ways) that are able to behave in a way that is very similar to micro-entities (Aerts et al 2000, Sassoli de Bianchi 2013a, Aerts and Sassoli de Bianchi 2014).

In that respect, one should demystify the usual belief that a quantum behavior would be only the prerogative of micro-entities, being instead a form of organization that can be found at different structural levels within our reality (Aerts and Sozzo 2015; Aerts and Sassoli de Bianchi 2018). Of course, it is at the micro-level that this organization appears to manifest itself in the most remarkable way, thanks to the non-spatial nature of the micro-entities.

But also human concepts are genuine non-spatial entities, hence in the human conceptual realm their quantum-like behavior appears to be as fully explicated as that of the micro-entities, even though, of course, not all the remarkable symmetries that govern the physical microworld are also present in the much younger human conceptual domain.

Now, considering the huge success of quantum theory in modeling different cognitive situations, like those involving decision-making, conceptual reasoning, human memory and other cognitive phenomena, i.e., that human conceptual entities, when they interact with cognitive systems, appear to be very similar to the quantum entities interacting with measuring apparatuses, it became natural at some point, for one of the initiators of quantum cognition, Diederik Aerts, to ask and take very seriously the following question (Aerts 2010a, b):

If quantum mechanics as a formalism models human concepts so well, perhaps this indicates that quantum particles themselves are conceptual entities?

Aerts then formulated the following speculative hypothesis, which is today at the basis of the so-called conceptuality interpretation of quantum mechanics (Aerts 2010a, b):

The nature of a quantum entity is 'conceptual,' i.e., it interacts with a measuring apparatus (or with an entity made of ordinary matter) in an analogous way as a concept interacts with a human mind (or with an arbitrary memory structure sensitive to concepts).

In other words, according to Aerts' hypothesis, the elementary microscopic entities, which we know cannot be consistently described in terms of particles and waves (or even fields), would nevertheless behave as something very familiar to all of us, as we continually experience them in a very intimate and direct way: concepts (Aerts 2009, 2010a, 2010b, 2013).

To help understand why such a hypothesis makes sense, we have to explain that concepts, like physical systems, can be modeled as entities that can be in different states, where a state has to be generally understood as an expression of what an entity is, in terms of its actual and potential properties in a given moment (Aerts et al 2016), which 


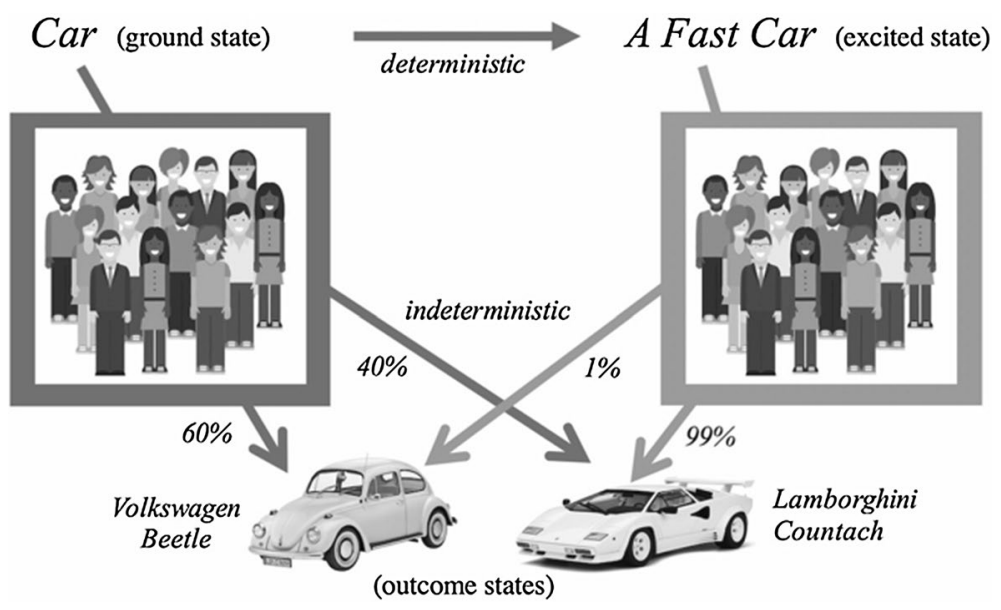

Fig. 13 Different states of the conceptual entity Car will produce different outcome probabilities, when subjected to a given interrogative context, here consisting in determining which one of the two more concrete (outcome) states, Volkswagen Beetle and Lamborghini Countach, better represent Car, when the latter is either in its 'ground state' or in the 'excited state' defined by the combination A Fast Car

can be described using different mathematical notions, depending on the specific formalism adopted. For instance, in quantum mechanics states are usually described by vectors belonging to a complex vector space, called Hilbert space.

The way a concept can change its state depends on the type of context with which it interacts. As a very simple example, consider the concept $\operatorname{Car}$ (we will use capital letters to distinguish abstract concepts from written words, which are the traces left by the latter on a given document). When considered in the context of itself, the conceptual entity Car can be said to be in its most neutral meaning state, sometimes referred to as the ground state of the concept. But it is also possible to combine the concept Car with other concepts. This is precisely what we humans typically do when we use our language: we combine concepts in order to create new meanings.

So, if Car is combined with Fast, say in the sentence A Fast Car, its state will not anymore be considered to be the ground state, but a different "excited" state. More precisely, when we go from Car to A Fast Car, the Car conceptual entity changes its state in a deterministic way. This is similar to what happens to the spin of a neutron when it passes through a magnetic field, also producing a deterministic change of its state that one can easily determine by solving the corresponding Schrödinger equation.

But to highlight the difference between two states, beyond considerations of a purely theoretical nature, one has to perform measurements, that is, one has to subject the conceptual entity to a given interrogative context, which in general will be indeterministic. For example, take two specific examples of cars, like a Volkswagen Beetle and a Lamborghini Countach. Then ask a group of people which one of the two best represents the more abstract concept Car. As it is not difficult to imagine, some people will choose the Volkswagen Beetle and others the Lamborghini Countach, and one can expect that both exemplars will be chosen with comparable frequencies, say $60 \%$ and $40 \%$, respectively (see Fig. 13).

Then take another group of people (or the same) and ask them the same question but this time in relation to A Fast Car. No doubts, almost all, if not all, will now select the 
Lamborghini Countach (see Fig. 13). In other words, the outcome probabilities will change dramatically when using A Fast Car instead of Car, i.e., when we consider different states of the conceptual entity. The same is true when performing a measurement in quantum mechanics: different states will produce different probability distributions in relation to a given set of possible outcomes.

Having said that, let us briefly describe some of the situations where the conceptuality interpretation allows one to better understand the strange behavior of the quantum microentities in a way that no other interpretations allows to do [for more details, we refer to Aerts (2009, 2010a, 2010b, 2013) and to the more recent review article Aerts et al (2018a, $\mathrm{b}, \mathrm{c})]$.

\subsection{Non-Spatiality}

Quantum entities are usually in non-spatial states because, being conceptual entities, they can be in states having different degrees of abstractness (or different degrees of concreteness), and only the most concrete (i.e., less abstract) states would correspond to those belonging to our spatial theater. For example, in the special case of human concepts, we can observe that the concept Thing, in its ground state, is undoubtedly more abstract than when in the state The Thing Is A Car, which in turn is more abstract than when in the state The Thing Is A Car Called Lamborghini Countach, which is more abstract than the state The Thing Is A Car Called Lamborghini Countach That Is Owned By My Neighbor. Clearly, this latter state of Thing brings the concept into close correspondence with the world of objects that belong to our three-dimensional space.

\subsection{Heisenberg Uncertainty Principle}

If quantum entities are conceptual, then they cannot be simultaneously maximally abstract and maximally concrete, which is none other than the uncertainty principle of Heisenberg rephrased in conceptual terms, now becoming perfectly self-evident. A neutron with a well-defined momentum would be a neutron in a maximally abstract state, whereas a neutron with a well-defined position would be a neutron in a maximally concrete state, and all states in between these two limit situations would be non-spatial states having an intermediary degree of abstractness (or of concreteness). In other words, there is a necessary tradeoff between abstractness and concreteness: the more we increase the former and the more the latter will decrease, and vice versa.

\subsection{Entanglement}

The mysterious non-spatial connections that are responsible for the creation of correlations in joint measurements, able to violate Bell's inequalities, would be nothing but connections through meaning. In other words, if the nature of the micro-entities is conceptual, then they are expected to spontaneously and systematically connect by sharing meaning, and since meaning connections are complex (multidimensional) abstract elements of our reality, this explains why they cannot be represented as simple spatial connections detectable in our three-dimensional theater. Note that Bell's inequalities can be easily violated when joint measurements are conducted in the psychological laboratories, on conceptual combinations that are adequately connected through meaning, thus giving further credit to 
the conceptuality interpretation of quantum entanglement; see for instance Aerts and Sozzo (2011) and Aerts et al (2018a, b).

\subsection{Indistinguishability}

Many conceptual entities, by combining with that particular category of concepts called numerals, will produce genuinely indistinguishable entities that still remain individuals. Hence, quantum indistinguishability becomes self-evident when quantumness is understood as an expression of conceptuality. Note that non classical (non-Maxwell-Boltzmann) statistics can be easily evidenced when considering certain combinations of words appearing in collections of documents.

Take for example the Ten Animals concept, which describes a collection of ten identical conceptual Animal entities. One can consider two possible states of Animal: The Animal Is A Cat (in short, Cat) and The Animal Is A Dog (in short, Dog). Then, one can perform counts, say on the Web, using a search engine like Google, to estimates the probabilities of finding these ten indistinguishable concepts in their different possible Cat and Dog states, like Eight Cats And Two Dogs, Seven Cats And Three Dogs, etc. Without going here into details, let us just mention that one finds in this way statistical behaviors that are quite similar to the Bose-Einstein one (with some added fluctuations), thus giving further credit to the conceptuality interpretation of quantum indistinguishability (Aerts, Sozzo and Veloz 2015; Aerts et al 2018a, b, c).

\subsection{Quantum Versus Classical}

According to the conceptuality interpretation, what we call objects are a limit situation of conceptual entities that can permanently remain in maximally concrete states. The best example of an object in the human conceptual realm or, to put it more precisely, of a concept that behaves similarly to an object, is what we call a story, i.e., a conceptual entity that is the result of a very large combination of different concepts all connected together through the "meaning fabric" of a specific narrative.

Without entering into the details, let us just mention the following interesting observation. Within the conceptual realm, concepts can be meaningfully combined using both the "and" and "or" logical connectives. If $A$ and $B$ are two concepts, then $A$ And $B$ and $A$ Or B are also bona fide concepts. On the other hand, if A and B are two objects, then although 'A and B' can still be considered to be an object (the composite object formed by the combination of object A and object B), 'A or B' cannot be associated anymore with any object, but only with a concept, and this is one of the fundamental differences between concepts and objects.

The situation is similar for stories. In our human cultural landscape, we can find many stories that are of the form 'A and B', even when A and B are very long and complex stories. As an example, think of book series, which are big composite stories of the form 'A and B and C..." On the other hand, if A and B are two full-fledged stories, 'A or B' will usually not be associated with a genuine (meaningful) story within our human culture. Hence, stories behave similarly to objects and the notion of story allows one to understand how certain typologies of conceptual entities, formed by the combination of numerous elementary concepts, end up behaving in ways that are similar to the way objects behave.

For a further discussion of this subtle question, about the distinction between concepts and objects, see for instance Aerts et al (2018a, b, c) and the references cited therein. 


\subsection{Open Problems in Physics}

The conceptuality interpretation offers interesting insights into many open problems of modern physics, like the measurement problem, quark confinement, the existence of different generations of elementary particles, dark matter, the lack of evidence for supersymmetry, etc. For the exploration of these interesting issues, we refer the interested reader to (Aerts 2009, 2010a, 2010b, 2013; Aerts et al 2018a, b, c).

\section{Conclusion and Perspectives}

We started this essay by referring to Plato's and Abbott's allegories, suggesting that our spatial theater would be the expression of a very limited perspective. By means of some examples, taken from our investigation of the micro-world, we tried to make the case that there is some deep truth in these allegories. There is however also an important aspect that the latter were not able to capture, which is the following. When the higher-dimensional quantum entities are viewed from the limited perspective of our spatial classical representation, the process is never amenable to just an act of discovery. This is so because quantum observations, apart exceptional circumstances, cannot be understood as mere processes of discovery of pre-existing properties, but literally as processes of creation of properties that were just potentially existing prior to the observational process. To put it differently, when the higher-dimensional quantum realm is brought into manifestation within our spatial theater, the process is generally non-deterministic and of the symmetry breaking kind, i.e., a process where the actual breaks the symmetry of the potential (Aerts ans Sassoli de Bianchi 2017).

We also emphasized that our ancient construction of a spatial theater resulted from our perception of the macroscopic objects of our environment, mediated by our physical senses, particularly the working together - in a compatible way-of our senses of sight and touch (Aerts 2014). However, when using more sophisticated measuring instruments in controlled experimental conditions, we were able to deepen our perceptions and observe that the behavior of the micro-entities is extremely puzzling, as their full reality was impossible to represent within the confines of a single spatial representation. ${ }^{4}$

Thanks to the success of the emerging field of quantum cognition, it became however apparent that this strangeness of the quantum entities is probably due to the fact that we are using the wrong "image" when we try to capture their nature: we think of them in terms of objects instead of (non-human) concepts. In other words, if on one hand our senses have contributed to the illusion of a three-dimensional spatial world, formed by macroscopic objects, it is our more recent and abstract way of interacting with reality (more recent in terms of our evolution as a species on this planet), guided by language and meaning, which

\footnotetext{
${ }^{4}$ Note that we can find (more or less explicit) traces of this impossibility in the ontologies of the different realistic quantum interpretations. For example, in Kastner's possibilistic transactional interpretation, it received the name of pre-spacetime, or pre-empirical layer (Kastner 2013, Aerts \& Sassoli de Bianchi, 2017). In the Bohmian view, a related notion is that of the implicate (pre-spatiotemporal) order (Bohm 1957). And to give a last example, the infinite number of constantly branching spatial worlds of the manyworlds interpretation certainly cannot be represented within a single spatial representation, hence a many (spatial) worlds reality is again a non-spatial reality; see also the discussion in Aerts \& Sassoli de Bianchi (2015b).
} 
appears to be the one able to bring us closer to the deeper aspects of our reality, which are genuinely non-spatial and most probably conceptual in nature.

From the idea that the building blocks of our physical reality would be conceptual entities carrying meaning and exchanging it with the different pieces of ordinary matter, a natural pancognitivist view emerged (Aerts and Sassoli de Bianchi 2018). This is however not a view to be understood in an anthropomorphic way, as is clear that human cognition is a very recent episode of formation of a conceptual structure, which took "place" in the ambit of a much more ancient evolutionary process, where everything within reality would participate in cognition.

Note that the mathematical language of our physical theories needs to be always accompanied by a suitable network of physical concepts, used to coherently relate the different mathematical entities and provide meaning to the portion of reality these theories are aimed to represent and describe (De Ronde 2018). Following the hypothesis of the conceptuality interpretation, one might be tempted to believe that human concepts would then be able to describe reality precisely as it is. This, however, would be an incorrect way to understand the message of the conceptuality interpretation, which requires to properly distinguish the human conceptual layer from that of the physical entities. We can certainly use our human concepts to try to represent and understand the (non-human) meaning that is vehiculated by the micro-physical entities and their combinations, but this does not mean that the two conceptual layers can be considered to be equivalent as regards their meaning content.

In a sense, it is like learning a new language, belonging to an ancient alien culture about which we know nothing, as it developed in territories and times completely different from ours. Most of the concepts in the language of that extra-terrestrial culture will have no direct correspondence with ours, although this should not prevent us from trying to approximate their meaning by using suitable combinations of concepts that are today present in our language. However, nothing guarantees that our language will be sufficiently rich to faithfully represent every aspect of that alien culture, especially if the experiences and behaviors that gave rise to it are too different from those that gave rise to ours. In other words, generally speaking, when one language studies another language, there are no a priori reasons for the concepts contained in the former to coincide, or be similar, to those contained in the latter, particularly so if the two languages do not necessarily share the same origin.

In that respect, note also that in the conceptuality interpretation two lines of going from the concrete to the abstract are distinguished: a parochial line, which has more to do with the way we humans have abstracted concept from objects, in the course of our recent evolution on this planet, and what we believe is a more universal line, which is about observing how a large number of concepts can enter a more concrete state by combing in a meaningful way to form what we humans, in our culture, call "stories." These are of course subtle aspects of the conceptuality interpretation, still under investigation, which would require more detailed explanations, and for this we refer the reader to (Aerts et al 2018a, b, c) and the references cited therein.

Coming back to pancognitivism, such a view has of course consequences also for our understanding of evolution. Indeed, if the nature of the physical entities is fundamentally conceptual, and if conceptuality and quantumness are just different ways to outline a same nature, then we need to adopt a much larger-quantum-like and conceptuallike-perspective not only on reality, but also on the mechanisms governing evolution. More precisely, adopting a quantum-like perspective on evolution means to understand the Darwinian natural selection account not only as a process of selection of properties that are already actual, i.e., already expressed within our spatiotemporal environment, 
but more generally as a process of selection through the actualization of properties that only possess a potential status. In other words, evolution would be the result of more general forms of interaction than those usually considered, with the different evolutionary contexts exerting their influences (typically in a sequential manner) according to dynamics of the (weighted) symmetry breaking kind, where selection would be operated from a wider basin of possibilities (Gabora and Aerts 2005a,b; Aerts et al 2011; Aerts and Sassoli de Bianchi 2018).

Also, adopting a conceptual-like perspective means that the evolution of the different biological species would be much more like a cultural evolution. This means that our human culture, which appeared as a secondary evolutionary process following that of the biological species, would be part of a more ancient and primordial process of "cosmic cultural change," in force since the very beginning of our reality. This also means that it is not Darwinian evolution that should be considered the general model for cultural evolution, that is, for describing also epistemological and conceptual changes, but the other way around: it is cultural evolution, the processes of change happening at the conceptual, psychological and social levels around us, that might well represent our most advanced and general evolutionary model, also to be used to better understand our biological evolution as a species. Of course, we are not saying that the Darwinian evolutionary mechanisms would not apply as such, but only suggesting that they should be reframed in a larger conceptuality-like picture, in the same way that classical physics had to be reframed within the ampler frameworks of quantum mechanics and relativity theory (Aerts and Sassoli de Bianchi 2018).

As a closing thought, let us mention the Fermi paradox: the observation that intelligent life appears to be a rare phenomenon within our spatiotemporal theater, usually called the universe, despite of the fact that probabilistic estimates (for instance based on famous Drake's equation) would suggest the opposite. Of course, this could simply be due to the fact that the different forms of advanced intelligent life existing in the cosmos do not currently have great interest in being noticed by us, or simply that we have not taken sufficiently seriously, as a scientific community, the many unexplained sightings of presumed extraterrestrial (non-inert) objects of which the vast UFO literature is rich. But whatever the reason, we can also hypothesize that our three-dimensional material and spatial universe is in any case not the best place where to look for life and culture within our non-spatial reality. Quoting from (Aerts and Sassoli de Bianchi 2018):

Life and culture might indeed more abundantly be found not so much by exploring our universe in width, i.e., its spatial vastness, but in depth, i.e., exploring those regions that, from our spatiotemporal perspective, appear to be non-spatial and non-temporal, and in that sense more conceptual than objectual.

Remains the problem of learning how to explore reality not only in width (the outer space, as typically explored by the astronauts), but also in depth or, better, how to further the "in depth exploration" that we have just initiated. Are we condemned to contemplate the much broader non-spatial reality by remaining forever confined within our three-dimensional spatial theater, that is to say, by only taking a peek through the quantum and relativistic windows, without ever stepping over it, or will we be able one day to unlock new possibilities and promote actual "inner space" explorations? This is a question to which it is impossible to provide any satisfactory answer today, but on which it is certainly possible and useful to meditate. 
Acknowledgements The author thanks Ulrike M. Bohlmann for having provided the stimulus of writing this article. He also thanks Diederik Aerts, Tomas Veloz and two anonymous reviewers, for their useful and pertinent comments, which have certainly improved the content of this article.

Open Access This article is licensed under a Creative Commons Attribution 4.0 International License, which permits use, sharing, adaptation, distribution and reproduction in any medium or format, as long as you give appropriate credit to the original author(s) and the source, provide a link to the Creative Commons licence, and indicate if changes were made. The images or other third party material in this article are included in the article's Creative Commons licence, unless indicated otherwise in a credit line to the material. If material is not included in the article's Creative Commons licence and your intended use is not permitted by statutory regulation or exceeds the permitted use, you will need to obtain permission directly from the copyright holder. To view a copy of this licence, visit http://creativecommons.org/licenses/by/4.0/.

\section{References}

Abbott, E. A. (1884). Flatland: A romance in many dimensions. London: Seeley and Co.

Aerts, D. (1984). The missing elements of reality in the description of quantum mechanics of the EPR paradox situation. Helvetica Physica Acta, 57, 421-428.

Aerts, D., et al. (1990). An attempt to imagine parts of the reality of the micro-world. In J. Mizerski (Ed.), Problems in Quantum Physics II; Gdansk '89 (pp. 3-25). Singapore: World Scientific Publishing Company.

Aerts, D. (1999). The Stuff the World is Made of: Physics and Reality. In D. Aerts, J. Broekaert, E. Mathijs, \& K. Academic (Eds.), The white book of 'Einstein meets Magritte: An interdisciplinary reflection (pp. 129-183). Dordrecht: Springer.

Aerts, D. (2009). Quantum particles as conceptual entities: A possible explanatory framework for quantum theory. Foundations of Science, 14, 361-411.

Aerts, D. (2010a). Interpreting quantum particles as conceptual entities. International Journal of Theoretical Physics, 49, 2950-2970.

Aerts, D. (2010b). A potentiality and conceptuality interpretation of quantum physics. Philosophica, 83, $15-52$.

Aerts, D. (2011). Quantum theory and conceptuality: Matter, stories, semantics and space-time. arXiv preprint arXiv: 1110.4766

Aerts, D. (2013). La mecànica cuántica y la conceptualidad: Sobre materia, historias, semántica y espaciotiempo. Scientiae Studia, 11, 75-100.

Aerts, D. (2014). Quantum theory and human perception of the macro-world. Frontiers in Psychology, 5, 554.

Aerts, D., Aerts Arguëlles, J., Beltran, L., Geriente, S., Sassoli de Bianchi, M., Sozzo, S., \& Veloz, T. (2018a). Spin and wind directions I: Identifying entanglement in nature and cognition. Foundations of Science, 23, 323-335.

Aerts, D., Aerts Arguëlles, J., Beltran, L., Geriente, S., Sassoli de Bianchi, M., Sozzo, S., \& Veloz, T. (2018b). Spin and wind directions II: A Bell state quantum model. Foundations of Science, 23, 337-365.

Aerts, D., Aerts Arguëlles, J., Beltran, L., Geriente, S., Sassoli de Bianchi, M., Sozzo, S. \& Veloz, T. (2019). Quantum entanglement in physical and cognitive systems: a conceptual analysis and a general representation. arXiv:1903.09103s

Aerts, D., \& Aerts, S. (2004). Towards a general operational and realistic framework for quantum mechanics and relativity theory. In A. C. Elitzur, S. Dolev, \& N. Kolenda (Eds.), Quo vadis quantum mechanics? possible developments in quantum theory in the 21st century. Berlin: Springer.

Aerts, D., Aerts, S., Broekaert, J., \& Gabora, L. (2000). The violation of bell inequalities in the macroworld. Foundations of Physics, 30, 1387.

Aerts, D., Bundervoet, S., Czachor, M., D’Hooghe, B., Gabora, L., Polk, P., \& Sozzo, S., et al. (2011). On the foundations of the theory of evolution. In D. Aerts (Ed.), Worldviews, science and us: Bridging knowledge and its implications for our perspectives of the world. Singapore: World Scientific.

Aerts, D., Gabora, L., \& Sozzo, S. (2013). Concepts and their dynamics: A quantum-theoretic modeling of human thought. Topics in Cognitive Science, 5, 737-772.

Aerts, D., \& Sassoli de Bianchi, M. (2014). The extended bloch representation of quantum mechanics and the hidden-measurement solution to the measurement problem. Annals of Physics, 351, 975-1025.

Aerts, D., \& Sassoli de Bianchi, M. (2015a). Do spins have directions? Soft Computing, 21, 1483-1504. 
Aerts, D., \& Sassoli de Bianchi, M. (2015b). Many-measurements or many-worlds? a dialogue. Foundations of Science, 20(4), 399-427.

Aerts, D., \& Sassoli de Bianchi, M. (2017). Quantum measurements as weighted symmetry breaking processes: the hidden measurement perspective. International Journal of Quantum Foundations, 3, 1-16.

Aerts, D., \& Sassoli de Bianchi, M. (2018). Quantum perspectives on evolution. In S. Wuppuluri \& F. A. Doria (Eds.), The map and the territory: Exploring the foundations of science, thought and reality (pp. 571-595). Berlin: Springer.

Aerts, D., Sassoli de Bianchi, M., \& Sozzo, S. (2016). On the foundations of the brussels operational-realistic approach to cognition. Frontier in Physics, 4, 17. https://doi.org/10.3389/fphy.2016.00017

Aerts, D., Sassoli de Bianchi, M., Sozzo, S., \& Veloz, T. (2018). On the conceptuality interpretation of quantum and relativity theories. Foundations of Science. https://doi.org/10.1007/s1069 9-018-9557-z

Aerts, D., \& Sozzo, S. (2011). Quantum structure in cognition: Why and how concepts are entangled. In: International Symposium on Quantum Interaction (pp. 116-127). Springer, Berlin

Aerts, D. \& Sozzo, S. (2015). What is Quantum? Unifying Its Micro-physical and Structural Appearance. In: Atmanspacher, H. et al (eds.). Quantum Interaction. QI 2014, pp. 12-23. Lecture Notes in Computer Science, vol. 8951. Springer, Cham.

Aerts, D., Sozzo, S., \& Veloz, T. (2015). The quantum nature of identity in human thought: Bose-Einstein statistics for conceptual indistinguishability. International Journal of Theoretical Physics, 54, $4430-4443$.

Aerts, S. (2005). A realistic device that simulates the non-local PR box without communication. arXiv preprint quant-ph/0504171.

Aspect, A. (1999). Bell's inequality test: more ideal than ever. Nature, 398, 189-190.

Aspect, A., Grangier, P., \& Roger, G. (1982). Experimental realization of einstein-podolsky-rosen-bohm gedankenexperiment: a new violation of Bell's inequalities. Physical Review Letters, 49, 91.

Bancal, J.-D., Pironio, S., Acín, A., Liang, Y.-C., Scarani, V., \& Gisin, N. (2012). Quantum non-locality based on finite-speed causal influences leads to superluminal signalling. Nature Physics, 8, 867-870.

Bell, J. S. (1964). One the Einstein Podolsky Rosen paradox. In J. S. Bell (Ed.), Speakable and unspeakable in quantum mechanics (pp. 195-200). Cambridge: Cambridge University Press.

Bell, J. S. (1971). In: B. d'Espagnat (Ed.), Proceedings of the International School of Physics "Enrico Fermi," Course XLIX (Academic Press, New York), p. 171.

Bell, J. S. (1981). Bertlmann's socks and the nature of reality. Journal de Physiqu Colloques, 42(C2), C41-C62.

Bohm, D. (1957). Causality and chance in modern physics (p. 117). London: Routledge.

Busemeyer, J. R., \& Bruza, P. D. (2012). Quanntum models of cognition and decision. Cambridge: Cambridge University Press.

Cocciaro, B., Faetti, S., \& Fronzoni, L. (2011). A lower bound for the velocity of quantum communications in the preferred frame. Physics Letters A, 375, 379-384.

Coretti, S., Hänggi, E., \& Wolf, S. (2011). Nonlocality is transitive. Physical Review Letters, 107, 100402.

de Ronde, C. (2018). Quantum superpositions and the representation of physical reality beyond measurement outcomes and mathematical structures. Foundations of Science, 23(4), 621-648.

Eibenberger, S., Gerlich, S., Arndt, M., Mayor, M., \& Tüxen, J. (2013). Matter-wave interference of particles selected from a molecular library with masses exceeding 10000 amu. Physical Chemistry Chemical Physics: PCCP, 15, 14696.

Einstein, A., Podolsky, B., \& Rosen, N. (1935). Can quantum-mechanical description of physical reality be considered complete? Physical Review, 47, 777-780.

Gabora, L., \& Aerts, D. (2005). Evolution as context-driven actualisation of potential: toward an interdisciplinary theory of change of state. Interdisciplinary Science Reviews, 30, 69-88.

Gabora, L. \& Aerts, D. (2005b). Distilling the Essence of an Evolutionary Process and Implications for a Formal Description of Culture. In: Kistler, W. (ed.). Proceedings of Center for Human Evolution Workshop 4: Cultural Evolution, May 18-19, 2000. Bellevue, WA: Foundation for the Future.

Gerlich, S., Eibenberger, S., Tomandl, M., Nimmrichter, S., Hornberger, K., Fagan, P. J., et al. (2011). Quantum interference of large organic molecules. Nature Communications, 2, 263.

Haven, E., \& Khrennikov, A. Y. (2013). Quantum Social Science. Cambridge: Cambridge University Press.

Kastner, R. E. (2013). The transactional Interpretation of quantum mechanics: The reality of possibility. New York: Cambridge University Press.

Morier, D. M., \& Borgida, E. (1984). The conjunction fallacy: a task specific phenomena? Personality and Social Psychology Bulletin, 10, 243-252.

Piron, C. (1990). Mécanique quantique: Bases et applications. Lausanne: Presses polytechniques et universitaires romandes. 
Rauch, H., Zeilinger, A., Badurek, G., Wilfing, A., Bauspiess, W., \& Bonse, U. (1975). Verification of coherent spinor rotation of fermions. Physics Letters A, 54, 425-427.

Salart, D., Baas, A., Branciard, C., Gisin, N., \& Zbinden, H. (2008). Testing the speed of 'spooky action at a distance.' Nature, 454, 861-864.

Sassoli de Bianchi, M. (2013a). Using simple elastic bands to explain quantum mechanics: a conceptual review of two of Aerts' machine-models. Central European Journal of Physics, 11, 147-161.

Sassoli de Bianchi, M. (2013b). Quantum dice. Annals of Physics, 336, 56-75.

Sassoli de Bianchi, M. (2014). A remark on the role of indeterminism and non-locality in the violation of Bell's inequality. Annals of Physics, 342, 133-142.

Sassoli de Bianchi, M. (2017). Theoretical and conceptual analysis of the celebrated $4 \pi$-symmetry neutron interferometry experiments. Foundations of Science, 22, 627-653.

Schrödinger, E. (1935). Naturwissenschaftern, 23, 807. English translation. In: John D. Trimmer (1980). Proceedings of the American Philosophical Society 124, p. 323. Reprinted in: J. A.

Tversky, A., \& Kahneman, D. (1983). Extension versus intuitive reasoning: The conjunction fallacy in probability judgment. Psychological Review, 90(4), 293-315.

Vigier, J. P., Dewdney, C., Holland, P. R., \& Kyprianidis, A. (1987). Causal particle trajectories and the interpretation of quantum mechanics. In B. J. Hiley \& F. D. Peat (Eds.), Essays in honour of David Bohm (pp. 169-204). London: Routledge.

Wendt, A. (2015). Quantum mind and social science. Cambridge: Cambridge University Press.

Werner, S. A., Colella, R., Overhauser, A. W., \& Eagen, C. F. (1975). Observation of the phase shift of a neutron due to precession in a magnetic field. Physical Review Letters, 35, 1053.

Wheeler, J. A., \& Zurek, W. H. (1983). Quantum theory and measurement. Princeton: Princeton University Press.

Zou, X. Y., Grayson, T., Wang, L. J., \& Mandel, L. (1992). Can an "empty" de Broglie pilot wave induce coherence? Physical Review Letters, 68, 3667-3669.

Publisher's Note Springer Nature remains neutral with regard to jurisdictional claims in published maps and institutional affiliations.

Massimiliano Sassoli de Bianchi graduated in physics from the University of Lausanne (UNIL), Switzerland, in 1989. From 1990 to 1991, he was an Assistant in the Department of Theoretical Physics (DPT) of the University of Geneva (UNIGE). In 1992, he joined the Institute of Theoretical Physics (IPT) at the Federal Institute of Technology in Lausanne (EPFL), where he received the Ph.D. degree in physics in 1995. Since 1996, he has been working in the private sector and as an independent researcher. In 2016, he joined the Center Leo Apostel for Interdisciplinary Studies (CLEA), at the Vrije Universiteit Brussel (VUB), as a research fellow. He is also the director of the Laboratorio di Autoricerca di Base (LAB), in Lugano, Switzerland, and the Editor of the Italian journal AutoRicerca. His research activities are focused on the foundations of physics, quantum cognition and consciousness studies. 